\title{
Thermophysical Properties of Mixtures of Thorium and Uranium Nitride
}

\author{
S.S. PARKER $@{ }^{1,3}$ S. NEWMAN, ${ }^{2}$ A.J. FALLGREN, ${ }^{2}$ and J.T. WHITE ${ }^{1}$ \\ 1.-Los Alamos National Laboratory, Materials Science and Technology Division, Los-Alamos, \\ USA. 2.-Los Alamos National Laboratory, Nuclear Engineering and Non-Proliferation \\ Division, Los Alamos, USA. 3.—e-mail: sparker@lanl.gov
}

The miscibility, lattice parameter, and thermophysical properties of $\left(\mathrm{Th}_{0.2} \mathrm{U}_{0.8}\right) \mathrm{N}$ and $\left(\mathrm{Th}_{0.5} \mathrm{U}_{0.5}\right) \mathrm{N}$ have been investigated. It is shown that additions of thorium nitride (ThN) to uranium nitride (UN) increases the thermophysical performance of the mixed nitride fuel form in comparison to reference UN. In the more dilute limit, additions of ThN serve as a burnable neutronic poison and reduces the change in $k_{\text {eff }}$ over the lifecycle of the fuel. At higher concentrations, additions of ThN serve as a significant fertile source of ${ }^{233} \mathrm{U}$. Where appropriate, comparisons to previous work on $\mathrm{UN}+\mathrm{PuN}$ mixtures are made, as this is a comparable fuel form for potential fast reactor concepts, and a suitable point of contrast in the possible design space afforded by mixed $\left(\mathrm{Th}_{\mathrm{x}} \mathrm{U}_{1-\mathrm{x}}\right) \mathrm{N}$ fuel forms. The data from this work are the input parameters for finite element modeling of the temperature distribution in a compact reactor. The results of modeling and simulation of this core design are shown for the case of steady-state operation and during double, adjacent heat pipe failure.

\section{INTRODUCTION}

Thermal conductivity, which is highly dependent on temperature, chemistry, and crystal structure, is an important factor of nuclear fuel performance under irradiation. The majority of the energy produced during fission is deposited as heat in the fuel, and this energy must dissipate by thermal conduction. Heat retention near the centerline, a problem in oxide fuels which exhibit low thermal conductivity that decreases with increasing temperature, contributes to deformation and cracking of the fuel over time. Ultimately, the onset of melting at the centerline of the fuel pellet limits the power density of the core. Changing from $\mathrm{UO}_{2}$ to non-oxide ceramic nuclear fuels allows the selection of a fuel architecture based on the desired chemistry, material properties, and irradiation behavior. The field of non-oxide nuclear fuels generally concerns the study of borides, carbides, nitrides, aluminides, and silicides of uranium, plutonium, and, to a lesser

(Received May 22, 2021; accepted August 11, 2021; published online September 9, 2021) extent, thorium. While some of these data exist for many of these compounds, these datasets do not offer a comprehensive description of the fuel characteristics as a function of temperature, time, and irradiation. Additionally, little is known of many non-oxide thorium ceramic compounds, or the properties of mixtures of these actinides.

Changes in chemistry from oxide to nitride ceramic nuclear fuel are associated with trade-offs in various intrinsic material properties. Generally, a departure from oxides results in a marginally lower melting point, but significantly higher thermal conductivity. For the case of fixed linear power density from fission across the fuel, and for the same period of fuel burnup, such an increase in thermal conductivity results in reduced centerline temperatures and a reduction in the thermal gradient from centerline to surface. Fuel forms which may retain a higher thermal conductivity as a function of burnup will improve fuel performance by mitigating temperature-dependent degradation by fuel swelling, grain growth, and fission gas release. The low thermal conductivity of actinide oxides as a function of temperature and density is well studied, but the physical origin of this limited thermal transport is 
not well understood. ${ }^{1-3}$ Recent modeling efforts theorize that large anharmonicity in the optical modes of phononic conduction in the oxide lattice of $\mathrm{UO}_{2}$ and $\mathrm{PuO}_{2}$ results in the low thermal conductivity of these materials. ${ }^{1,4}$ In the study by Yin and Savrasov, ${ }^{4}$ the calculated phononic dispersions indicated that lattice vibrations associated with out-ofphase displacements of oxygen were the dominant contributor to phononic dampening, and that this effect increases with increasing temperature. The nitrogen bound in $\mathrm{UN}$ and PuN, by contrast, must therefore either exhibit lower magnitude displacements that are out-of-phase or higher order vibrations which are in-phase. This effect, especially in $\mathrm{UN}$, is found to persist even after electronic contributions to thermal transport from 5 f electrons are considered. ${ }^{5}$ The removal of the dampening effect of oxygen is substantial: the thermal conductivity of $\mathrm{UN}$ is 4-8 times greater than that of $\mathrm{UO}_{2}$ in the temperature range relevant to the steady-state operation of a nuclear reactor. Previous investigations have suggested that $\mathrm{ThN}$ has a thermal conductivity at room temperature in the range of $40-50 \mathrm{~W} /(\mathrm{mK}){ }^{6-8}$ Given this result, additions of ThN in UN could significantly improve the thermal properties of UN.

Nuclear fuels are subjected to extreme thermomechanical conditions. Determination of the elastic properties as a function of temperature allows estimation of the fuel performance on startup, in steady-state, and during transient conditions. The hypothetical lifetime of nuclear fuel is predicated on the fissile inventory and the integrity of the fuel; however, the attainable core design lifetime is more practically limited by degradation of the cladding and the cladding performance under transient conditions. Additionally, mechanical degradation of the fuel due to the severe temperature and irradiation effects is unavoidable, and may contribute to limitations of reactor performance or the safety basis of the design. Fuel degradation has been well studied in $\mathrm{UO}_{2}$, which is known to form large radial cracks at high burnup which diminish fuel performance. ${ }^{9}$ Switching to a nitride architecture may improve the thermal stress resistance of the fuel, which may in turn improve in-pile performance. The property of UN to rapidly dissipate heat at elevated temperatures has been suggested to substantially reduce thermal shock and fuel cracking, which may occur on startup or during a rapid, transient increase in reactor power. ${ }^{10,11}$ Modeling elastic strain requires measurement of mechanical properties. However, elastic property measurement as a function of temperature, porosity, and burnup have not been conducted on UN or mixed $\left(\mathrm{Th}_{\mathrm{x}} \mathrm{U}_{1-\mathrm{x}}\right) \mathrm{N}$ fuels.

Following recent work on the characterization of UN and ThN, this study extends our research to mixed $\left(\mathrm{Th}_{\mathrm{x}} \mathrm{U}_{1-\mathrm{x}}\right) \mathrm{N}$ ceramics. ${ }^{12}$ In what follows, two compositions of $\left(\mathrm{Th}_{\mathrm{x}} \mathrm{U}_{1-\mathrm{x}}\right) \mathrm{N}$ were studied: $x=0.2$ and $x=0.5$. In the more dilute limit, $x=0.2$, thorium serves as a burnable neutronic poison and reduces the change in $k_{\text {eff }}$ over the lifecycle of the fuel, while simultaneously providing significant increases in the thermophysical properties of the mixed nitride fuel form. At this composition, the relative enrichment of fissile isotopes of uranium (i.e., ${ }^{233} \mathrm{U}$ or ${ }^{235} \mathrm{U}$ in a matrix of ${ }^{238} \mathrm{U}$ ) may be assumed to be low enriched (19.7\%) or high enriched $(>20 \%)$. In the more thorium-rich composition, $x=0.5$, higher uranium enrichment may be necessary in order to extend the operational lifetime of the reactor design. These compositions, in addition to the available data of the thermophysical and mechanical properties of pure ThN and UN, enable a description of the properties of arbitrary mixtures of ThN and UN. The miscibility, lattice parameter, and thermophysical properties are presented. Where appropriate, comparisons to previous work on UN + PuN mixtures are made, as this is a comparable fuel form for potential fast reactor concepts and a suitable point of contrast in the possible design space afforded by mixed $\left(\mathrm{Th}_{\mathrm{x}} \mathrm{U}_{1-\mathrm{x}}\right) \mathrm{N}$ fuel forms. x-ray diffraction techniques were used to measure the lattice parameters of the as-sintered mixtures. The measured thermophysical properties of $\left(\mathrm{Th}_{0.2} \mathrm{U}_{0.8}\right) \mathrm{N}$ and $\left(\mathrm{Th}_{0.5} \mathrm{U}_{0.5}\right) \mathrm{N}$ as a function of temperature are summarized. The data from this work are the input parameters for finite element modeling of the temperature distribution in a modified Megapower ${ }^{\odot}$ core design (Patent No. US $20160027536 \mathrm{~A} 1$ ), which is used as an illustrative example of the application of these fuel forms. A render of the cross section of this model is shown in Fig. 1a, and the finite element model of the steady state thermal profile calculated from the experimental data presented in this study is shown in Fig. $1 b$.

\section{EXPERIMENT}

\section{Mixing and Miscibility of $\left(\operatorname{Th}_{\mathbf{x}} \mathrm{U}_{1-\mathrm{x}}\right) \mathbf{N}$}

Homogenous mixing of $\mathrm{ThN}$ and $\mathrm{UN}$ is an essential requirement of a mixed fuel architecture. UN and $\mathrm{ThN}$ were produced by separate carbothermic reduction to nitridation (CTR-N) processes, and were subsequently mixed. Details concerning the synthesis methods to produce UN and ThN are described in Refs. 12 and 13. While not studied here, a mixed CTR-N starting with $\mathrm{ThO}_{2}+\mathrm{UO}_{2}+$ graphite may provide a readily scalable method for the production of mixed nitride powders. Starting with pure ThN and UN, co-milling produced sufficiently mixed feedstock. In order to reduce the risk of oxidation of sample material, powder processing and sintering occurred within an inert atmosphere glovebox where oxygen was monitored and remained below $30 \mathrm{ppm}$. A set of samples of $\left(\mathrm{Th}_{0.5} \mathrm{U}_{0.5}\right) \mathrm{N}$ and $\left(\mathrm{Th}_{0.2} \mathrm{U}_{0.8}\right) \mathrm{N}$ with dimensions suitable for measurement of thermophysical properties were produced by cold pressing and high-temperature sintering $(2000 \mathrm{~K})$ in flowing argon with $6 \%$ 


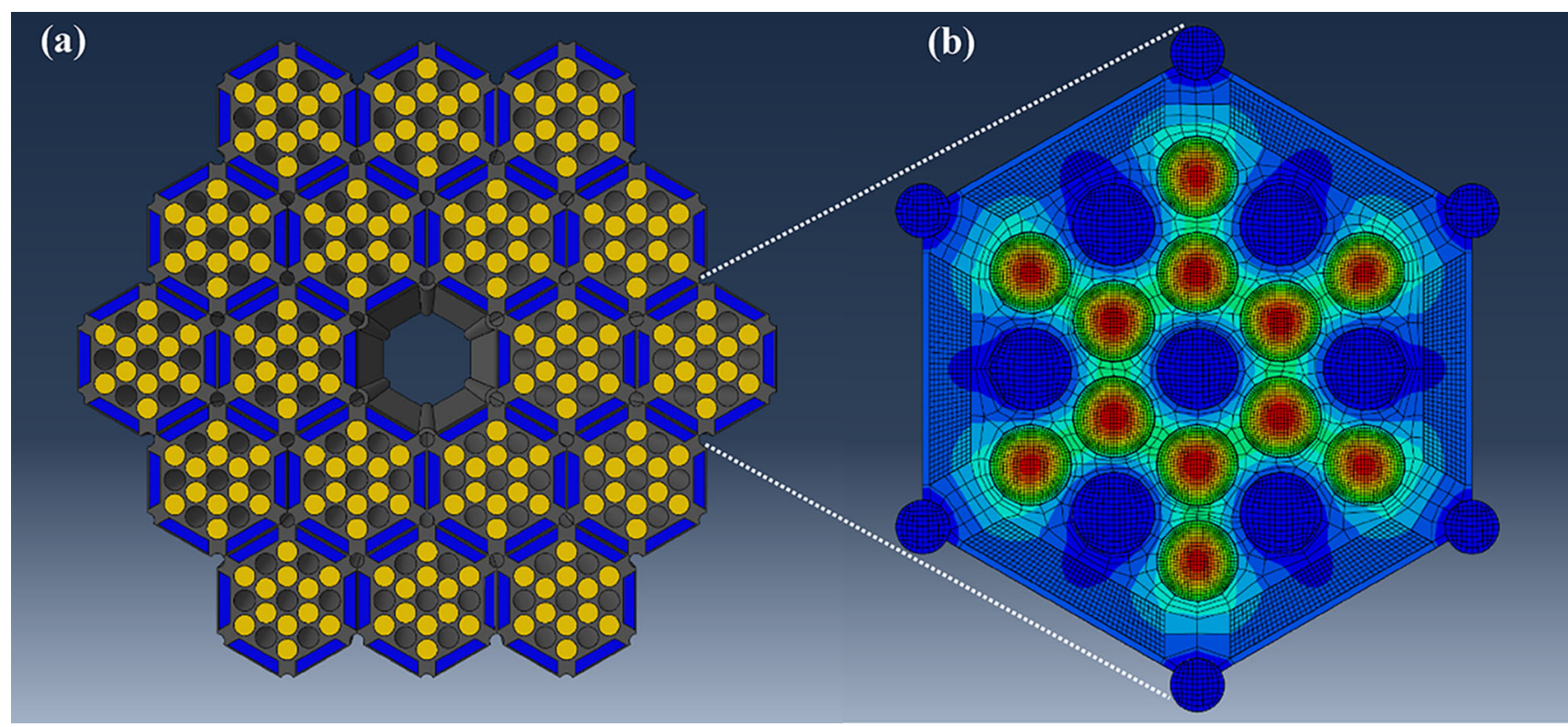

Fig. 1. (a) A render of the cross-section of the Monte Carlo N-Particle (MCNP) model of a compact reactor design. Gold zones indicate placement of the mixed $(T h x U 1-\times) \mathrm{N}$ fuel, the gray zone is the primary structural component, and blue zones are the moderator. (b) Given the power deposition calculated by the MCNP model, a finite element model was created for an individual fuel bundle. The colors shown are a heat map: the red zones are the center of the fuel pellets, where the majority of heat is deposited, and the dark blue zones are the coolant channels, which are fixed at $973 \mathrm{~K}$ as a boundary condition (Color figure online).

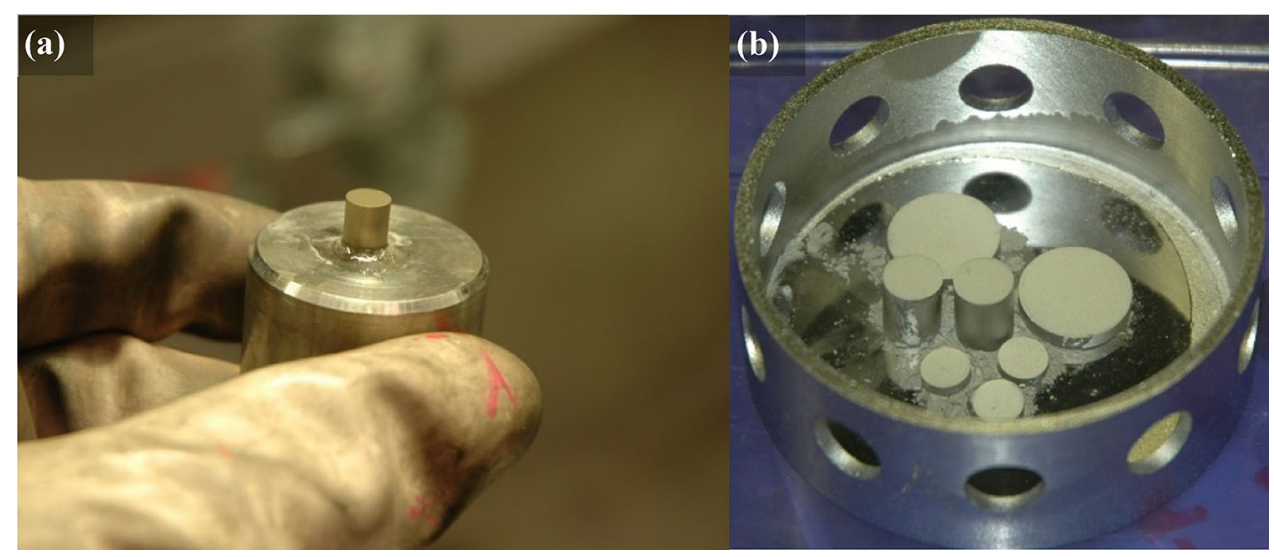

Fig. 2. (a) $\left(T h_{0.5} U_{0.5}\right) \mathrm{N}$, adhered to a polishing mount, and (b) $\left(\mathrm{Th}_{0.2} \mathrm{U}_{0.8}\right) \mathrm{N}$ monoliths on a powder bed of $\left(\mathrm{Th}_{0.2} \mathrm{U}_{0.8}\right) \mathrm{N}$ following high-temperature sintering (Color figure online).

hydrogen. Sample geometries were chosen according to the subsequent analytical method: samples for heat capacity measurement were $\sim 5.2 \mathrm{~mm} \times 1.5$ $\mathrm{mm}$, samples for thermal diffusivity measurement were $\sim 10 \mathrm{~mm} \times 2 \mathrm{~mm}$, and samples for measurement of the coefficient of thermal expansion were $\sim 5.2 \mathrm{~mm} \times 10 \mathrm{~mm}$. Tungsten liners and tungsten trays were utilized during all sintering steps. Figure 2 shows samples of each mixture following sintering: $\left(\mathrm{Th}_{0.5} \mathrm{U}_{0.5}\right) \mathrm{N}$ retains the characteristic gold color of $\mathrm{ThN}$, while $\left(\mathrm{Th}_{0.2} \mathrm{U}_{0.8}\right) \mathrm{N}$ exhibits the silver/gray color associated with UN. Samples were between 82 and $92 \%$ of the maximum theoretical density. Density measurement was confirmed by geometric and immersion techniques.

Dense pellets $(\sim 90 \%)$ were selected for measurement by x-ray diffraction (XRD); samples were processed into powder by $\mathrm{Al}_{2} \mathrm{O}_{3}$ mortar and pestle, and were enclosed within a sealed sample crucible prior to removal from the glovebox for x-ray analysis. Figure 3a shows the results of XRD scans on the mixed nitrides along with the data taken previously on pure ThN and UN. The mixtures are shown to be phase pure mononitrides with peaks shifted from the pure materials in proportion to composition of the sample. No oxide peaks are 


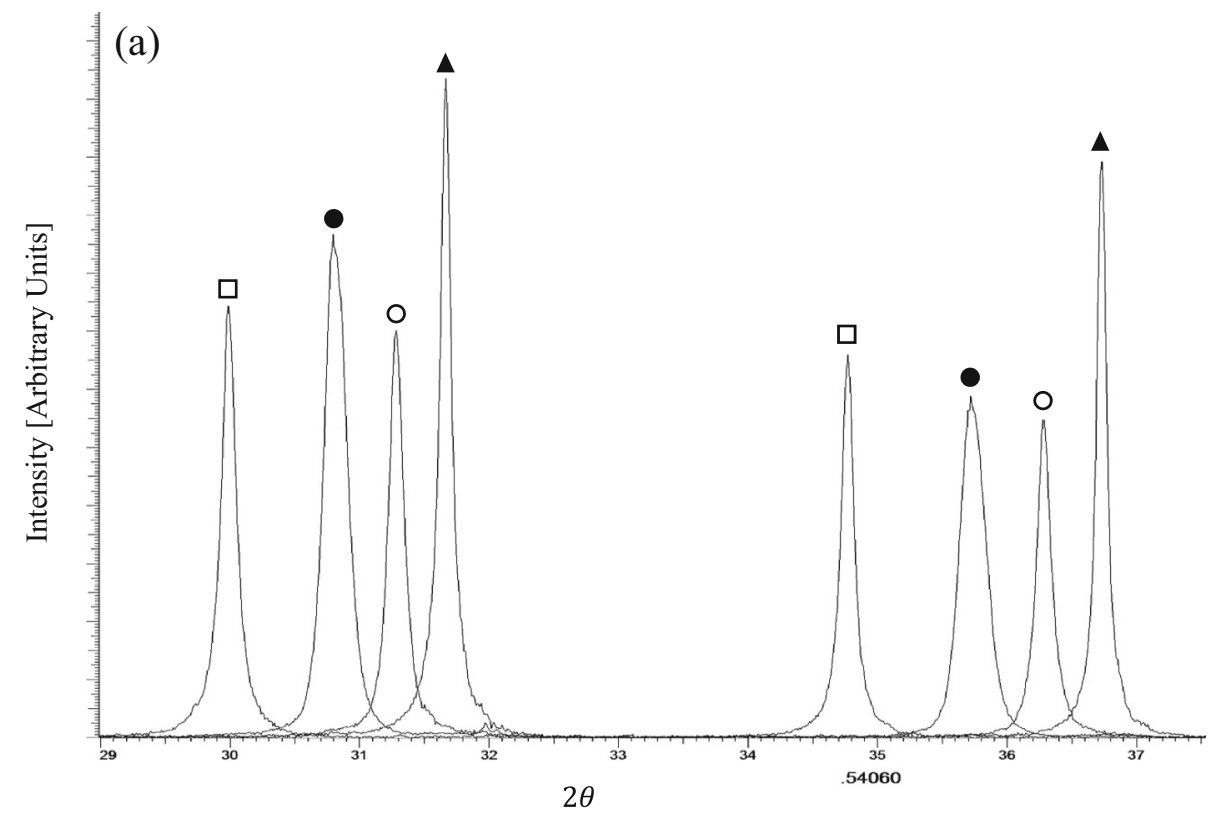

(b)

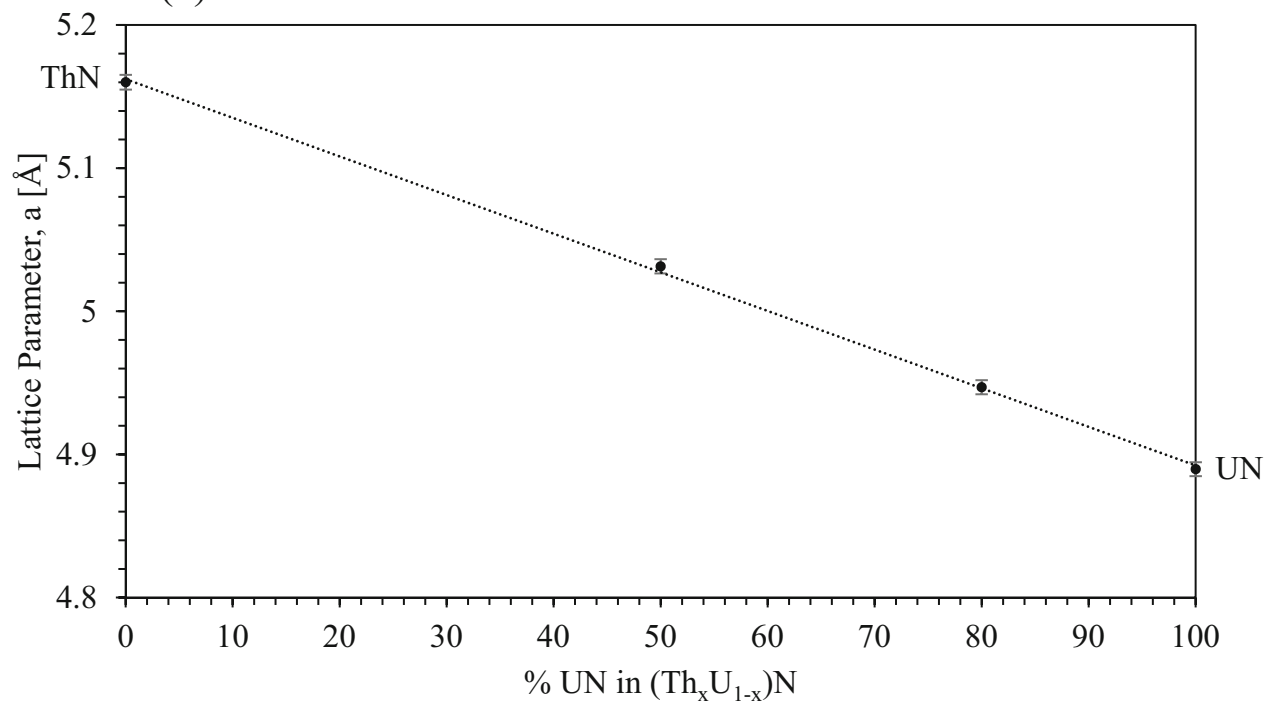

Fig. 3. (a) X-ray diffraction spectra for Square (ThN); Triangle (UN); Dark Circle $\left(\operatorname{Th}_{0.5} \mathrm{U}_{0.5}\right) \mathrm{N}$; and Light Circle $\left(T h_{0.2} \mathrm{U}_{0.8}\right) \mathrm{N}$. (b) Lattice parameter measurement of reference metallic $\mathrm{Si}, \mathrm{ThN}, \mathrm{UN},\left(\mathrm{Th}_{0.2} \mathrm{U}_{0.8}\right) \mathrm{N}$, and $\left(\mathrm{Th}_{0.5} \mathrm{U}_{0.5}\right)$. This plot shows the lattice parameter of the mixed nitrides and the pure materials. The solid solutions of ThN + UN follow Vegard's law.

visible in the XRD spectra. Implementing the lattice parameter measurement by the Bradley-Jay method, the lattice parameters of $\left(\mathrm{Th}_{0.5} \mathrm{U}_{0.5}\right) \mathrm{N}$ and $\left(\mathrm{Th}_{0.2} \mathrm{U}_{0.8}\right) \mathrm{N}$ were measured with respect to reference Si metal. The lattice parameter is determined from the measured lattice spacing as a function of $\cos ^{2}(2 \theta)$, and the lattice parameter of $\left(\mathrm{Th}_{\mathrm{x}} \mathrm{U}_{1-\mathrm{x}}\right) \mathrm{N}$ is plotted as a function of $x$ in Fig. 3b. It is shown that the lattice parameter is a linear combination of the lattice parameters of the pure materials. This finding is a demonstration of Vegard's law, and indicates that ThN and UN readily form a solid solution, for which the lattice parameter may be estimated as $a_{T h_{x} U_{1-x}}=x \cdot a_{T h N}+(1-x) \cdot a_{U N}$.
Formation of a solid solution enables use of conventional porosity correction models, which is discussed in "Finite Element Modeling of Thermal Profile: A Case Study" Section.

\section{Thermophysical Properties of $\left(\mathbf{T h}_{\mathbf{x}} \mathbf{U}_{1-\mathrm{x}}\right) \mathbf{N}$}

\section{Differential Scanning Calorimetry}

Heat capacity measurements were performed in a differential scanning calorimeter (DSC) (Pegasus 404C; Netzsch Instruments, Germany) equipped with platinum platforms, sample crucibles, and sample lids. The platinum crucibles were lined with $\mathrm{Al}_{2} \mathrm{O}_{3}$ trays in order to prevent reaction between 
ThN and Pt at elevated temperatures. The upper test limit of $1273 \mathrm{~K}$ was chosen in order to prevent interaction between the $\mathrm{Pt}$ trays and the $\mathrm{Pt}$ sensor head, which would result in damage to the sensor head. Specific heat capacity was determined by the ratio method, as described by ASTM standard E1269. Samples were ground to approximately plane parallel dimensions using a $15-\mu \mathrm{m}$ diamond polishing disk and were subsequently polished up to 1200 grit on $\mathrm{SiC}$ grinding paper. The sample height was set by the grinding process and chosen so as to match the volume of each sample with the volume of the available sapphire standards. The primary sources of error in this technique are the surface roughness of the side of the sample in contact with the tray, and the volume mismatch between the sample and the sapphire standard. Prior to running each experiment, the DSC chamber was twice purged with a turbo-molecular vacuum pump to a vacuum condition of at least $1 \times 10^{-5}$ mbar and refilled with ultra-high purity argon gas in order to reduce the potential for residual oxygen contamination at the onset of the experiment. A single sample measurement cycle consisted of running an identical profile four times consecutively: baseline check, sapphire standard measurements, sample measurements, and baseline verification. If no appreciable drift in the observed signal occurs between the first and second baseline, the sapphire and sample measurements are considered to be consistent.

\section{Dilatometry}

Samples were ground to approximately plane parallel dimensions using a $15-\mu \mathrm{m}$ diamond polishing disk; samples were ground to a fixed length so as to match calibration standards used by this technique. Measurement of sample thickness was taken as an average of 10 measurements by a digimatic indicator (543-783BCERT; Mitutoyo, Japan). A push rod dilatometer (DIL 402 CD; Netzsch Instruments) was used to measure the physical coefficient of thermal expansion $\alpha_{p}$, in a flowing inert (Ar) atmosphere. Prior to running the experiment, the dilatometer chamber was twice purged with a turbomolecular vacuum pump to a vacuum condition of at least $1 \times 10^{-4} \mathrm{mbar}$ and refilled with ultra-high purity argon in order to reduce the potential for residual oxygen contamination at the onset of the experiment. The apparatus was allowed to stabilize with a 1-h standby with flowing argon gas prior to each experiment. Using this experimental apparatus, the physical coefficient of thermal expansion $\alpha_{p}$ was measured up to $1300 \mathrm{~K}$ using a heating rate of $2.5 \mathrm{~K} / \mathrm{min}$. Eight $\alpha_{p}$ measurements on four unique samples were used to determine a standard error of $8 \%$.

\section{Laser Flash Analysis}

Cylindrical sample specimens for the determination of thermal diffusivity of $\mathrm{ThN}$ as a function of temperature were fabricated. Sample height was restricted to $(<3 \mathrm{~mm})$ so as to minimize radial thermal loss. Samples were ground to approximately plane parallel dimensions using a $15-\mu \mathrm{m}$ diamond polishing disk. Measurement of sample thickness was taken as an average of 10 measurements by the digimatic indicator. Prior to analysis, samples were coated in graphite (Graphit 33; Kintakt Chemie, Germany) in order to improve absorption of the laser pulse on the one side and emissivity of infrared blackbody radiation on the other. $\mathrm{Al}_{2} \mathrm{O}_{3}$ components was used within the LFA; no apparent reaction occurred between samples and the components. On the radiation-emitting side of the sample, a $10-\mathrm{mm}$ tungsten mask was used to fix the observable surface area. Throughout the experiment, flowing gettered argon process gas was utilized to control oxygen contamination. An in-line zirconia cell oxygen sensor (RapidOx OEM447; Cambridge Sensotec, Saint Ives, UK) was utilized to monitor oxygen contamination at the inlet and outlet of the LFA chamber. Oxygen levels remained below the limits of detection. A laser flash analyzer (LFA427; Netzsch Instruments) was utilized to determine thermal diffusivity. The test methodology follows that of ASTM E1461-13. Radiative loss from the sample is minimized due to the short time required to apply the pulse. The sample thermocouple was calibrated using the curie temperature of $\mathrm{Fe}$, from which a temperature uncertainty of $\pm 3 \mathrm{~K}$ is determined for measurements reported in this study. Data were collected both on heating and cooling, in 100-K increments and offset by $50 \mathrm{~K}$ while cooling. Three laser shots were recorded at each temperature condition. Reported diffusivity values were determined by fitting the temperature rise signal with a pulse-corrected Cape-Lehman model.

\section{RESULTS}

Thermophysical properties, including heat capacity, coefficient of thermal expansion, and thermal diffusivity were measured in a flowing high purity argon environment as a function of temperature up to $1700 \mathrm{~K}$. Thermal conductivity $(\lambda)$ as a function of temperature is then calculated from density $(\rho)$, heat capacity $\left(C_{P}\right)$, and thermal diffusivity $(D)$. The theoretical density at room temperature, $\rho_{o}$, is determined from the lattice parameter measurements.

\section{Heat Capacity}

The measured heat capacity of $\left(\mathrm{Th}_{0.5} \mathrm{U}_{0.5}\right) \mathrm{N}$ and $\left(\mathrm{Th}_{0.2} \mathrm{U}_{0.8}\right) \mathrm{N}$ are plotted with a linear extrapolation to $1700 \mathrm{~K}$ in Fig. $4 \mathrm{a}$ and b, respectively. The KoppNeumann rule, given by Eq. 1, was used to estimate the anticipated heat capacity of the mixtures: 

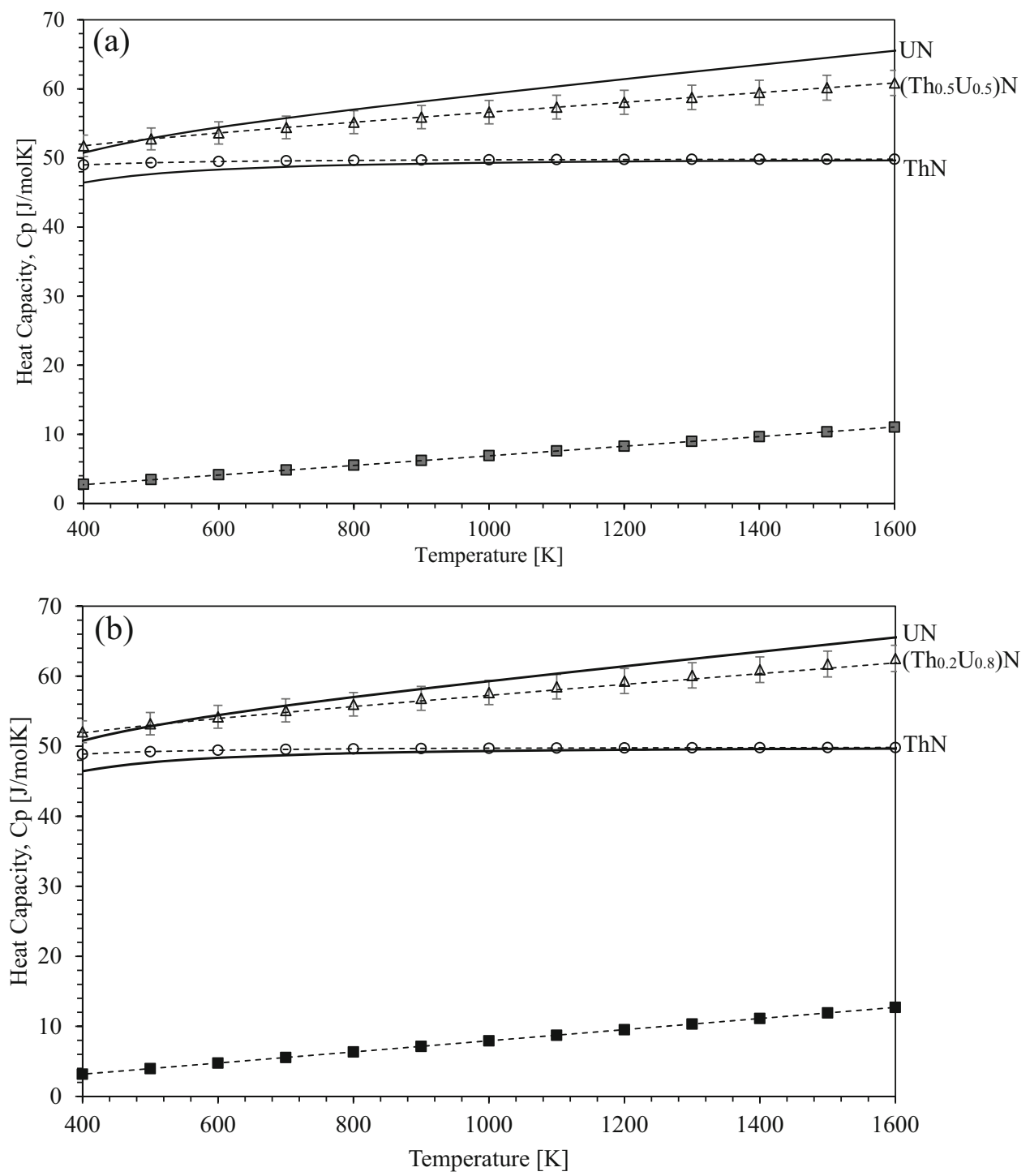

Fig. 4. Heat capacity of (a) $\left(\mathrm{Th}_{0.5} \mathrm{U}_{0.5}\right) \mathrm{N}$, and (b) $\left(\mathrm{Th}_{0.2} \mathrm{U}_{0.8}\right) \mathrm{N}$. The experimental results (Triangle) are plotted with $3 \%$ error. The measured heat capacity is separated into the constituent electronic contribution (Square) and the phononic contribution (Circle) for each case.

$$
C=\sum_{i=1}^{N}\left(C_{i} f_{i}\right)
$$

where $\mathrm{N}$ is the number of species in the mixture, $f_{i}$ is the relative fraction of specie $i$, and $C_{i}$ is the heat capacity of specie $i$. Both $C$ and $C_{i}$ scale with temperature, and the contributions from electronic and phononic transport may be considered separately. Available heat capacity data for UN as a function of temperature up to $1700 \mathrm{~K}$ are presented in comparison to $\mathrm{ThN}$ in order to enable a discussion of electronic versus phononic contributions to thermal transport in these materials. While the value of heat capacity as a function of temperature is found to be similar in magnitude in ThN and UN, notable differences arise and are attributed to differences in the proportion of thermal transport carried by electrons. While the theoretical prediction of heat capacity is in reasonable agreement with experimental data at high temperatures $(>1700 K)$, the model underestimates the heat capacity at room temperature by over $10 \%$ for both mixtures. Interestingly, this seems to reflect that the electronic contribution from the 5 f electrons in UN do not necessarily scale linearly in proportion to the atom fraction of $\mathrm{U}: \mathrm{Th}$ in $\left(\mathrm{Th}_{\mathrm{x}} \mathrm{U}_{1-\mathrm{x}}\right) \mathrm{N}$. While this effect is small, it might imply partial hybridization of metal-nitrogen bonding throughout the lattice. Measurement of the heat capacity of dilute additions on $\mathrm{UN}$ in $\mathrm{ThN}$ (i.e., $\mathrm{x}>0.8$ ) may provide additional insight into the nature and contribution of electronic transport to thermal conduction in UN. From the measured heat capacity of $\left(\mathrm{Th}_{0.2} \mathrm{U}_{0.8}\right) \mathrm{N}$ and $\left(\mathrm{Th}_{0.5} \mathrm{U}_{0.5}\right)$, the empirical equations of fit $(+/-$ $4 \%$ ) for heat capacity are given by Eqs. 2 and 3, respectively: 


$$
\begin{aligned}
C_{p,\left(T h_{0.2} U_{0.8} N\right.}= & 8.7 \cdot 10^{-3} \cdot T \\
& +6 N_{A} k_{B}\left(\frac{246.2}{T}\right)^{2} \frac{e^{246.2 / T}}{\left(e^{246.2 / T}-1\right)^{2}} \\
C_{p,\left(T h_{0.5} U_{0.5}\right) N}= & 7.5 \cdot 10^{-3} \cdot T \\
& +6 N_{A} k_{B}\left(\frac{198.3}{T}\right)^{2} \frac{e^{198.3 / T}}{\left(e^{198.3 / T}-1\right)^{2}}
\end{aligned}
$$

The measured heat capacity of ThN, UN, $\left(\mathrm{Th}_{0.2} \mathrm{U}_{0.8}\right) \mathrm{N}$, and $\left(\mathrm{Th}_{0.5} \mathrm{U}_{0.5}\right) \mathrm{N}$ are shown in Fig. $4 \mathrm{a}$ and $\mathrm{b}$. The $\mathrm{Cp}$ of $\left(\mathrm{Th}_{0.2} \mathrm{U}_{0.8}\right) \mathrm{N}$ agrees well with the theoretical estimate and displays a strong dependence on electronic contributions, as is the case in pure UN. The data for $\left(\mathrm{Th}_{0.5} \mathrm{U}_{0.5}\right) \mathrm{N}$ is slightly higher than what might be expected from a simplistic rule-of-mixtures calculations, which might suggest a slightly stronger electronic contribution. This effect is minor, however, and is within the reported error. It seems that the heat capacity of any arbitrary mixture may be calculated from the molar fractions and heat capacities of the pure components, to within an error of a few percent. Given that the heat capacity is essentially linear at high temperatures, extrapolation above $1600 \mathrm{~K}$ should not significantly amplify uncertainty.

\section{Coefficient of Thermal Expansion}

The average coefficient of thermal expansion from the complete dataset is plotted with reasonable linear extrapolation as a function of temperature up to $1500 \mathrm{~K}$ in Fig. 5. Theoretical estimates from the rule-of-mixtures are included as dashed lines; the data are in reasonable agreement with the predictions. The data for $\left(\mathrm{Th}_{0.2} \mathrm{U}_{0.8}\right) \mathrm{N}$ are slightly higher than the theoretical estimate, but are within the error of the method. The equations of fit for the coefficient of thermal expansion are given by Eqs. 4 and 5 in units of $[1 / \mathrm{K}]$ :

$$
\begin{aligned}
& \alpha_{\left(T h_{0.2} U_{0.8}\right) N}=1.62 \cdot 10^{-9} \cdot T+6.68 \cdot 10^{-6} \\
& \alpha_{\left(T h_{0.5} U_{0.5}\right) N}=2.67 \cdot 10^{-9} \cdot T+5.34 \cdot 10^{-6}
\end{aligned}
$$

\section{Thermal Diffusivity}

Thermal diffusivity was measured by laser flash analysis. The average measurement of thermal diffusivity on $\left(\mathrm{Th}_{0.2} \mathrm{U}_{0.8}\right) \mathrm{N}$ and $\left(\mathrm{Th}_{0.5} \mathrm{U}_{0.5}\right) \mathrm{N}$ is plotted with reasonable extrapolation as a function of temperature up to $1500 \mathrm{~K}$ in Fig. 6: the data plotted are as measured, without porosity correction. The porosity dependence of the thermal diffusivity of UN has been well studied, and such empirical corrections have been applied for the LFA samples with measured density as low as $82-84 \% .{ }^{14}$ The uncertainty reported in Fig. 6 is considered conservative in order to account for a lack of knowledge of the porosity dependence of $\mathrm{ThN}$ within the mixtures. While not within the scope of this investigation, the state of knowledge of mixed $\left(\mathrm{Th}_{\mathrm{x}} \mathrm{U}_{1-\mathrm{x}}\right) \mathrm{N}$ fuels would greatly benefit from a systematic study of the effects of sample porosity on thermal diffusivity in both ThN and in mixtures of ThN and UN. For this study, it is assumed that ThN and UN exhibit

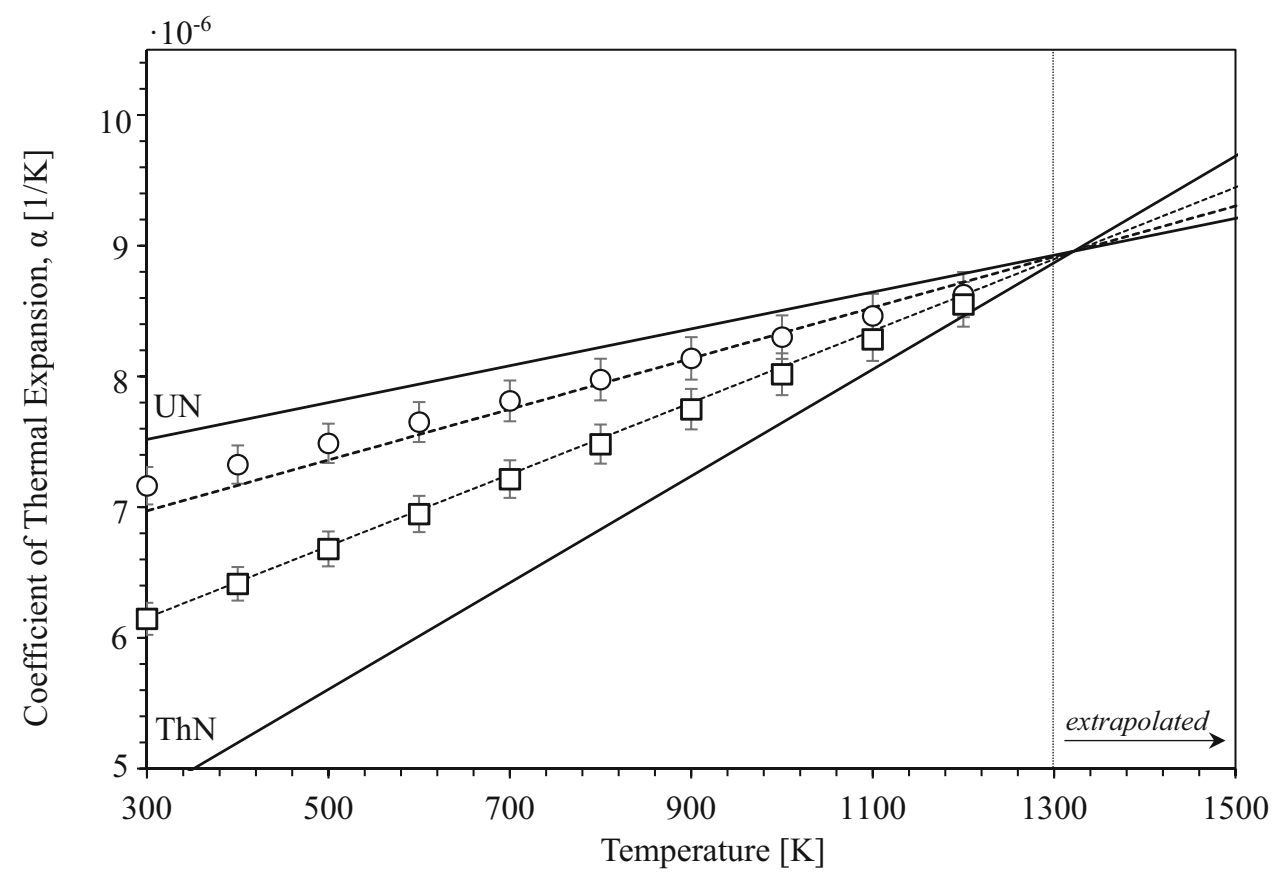

Fig. 5. Coefficient of thermal expansion of $\left(\mathrm{Th}_{0.2} \mathrm{U}_{0.8}\right) \mathrm{N}\left(\right.$ Circle) and $\left(\mathrm{Th}_{0.5} \mathrm{U}_{0.5}\right) \mathrm{N}($ Square), plotted against theoretical estimates. Data are plotted

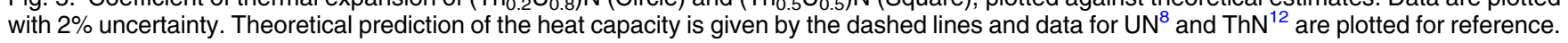




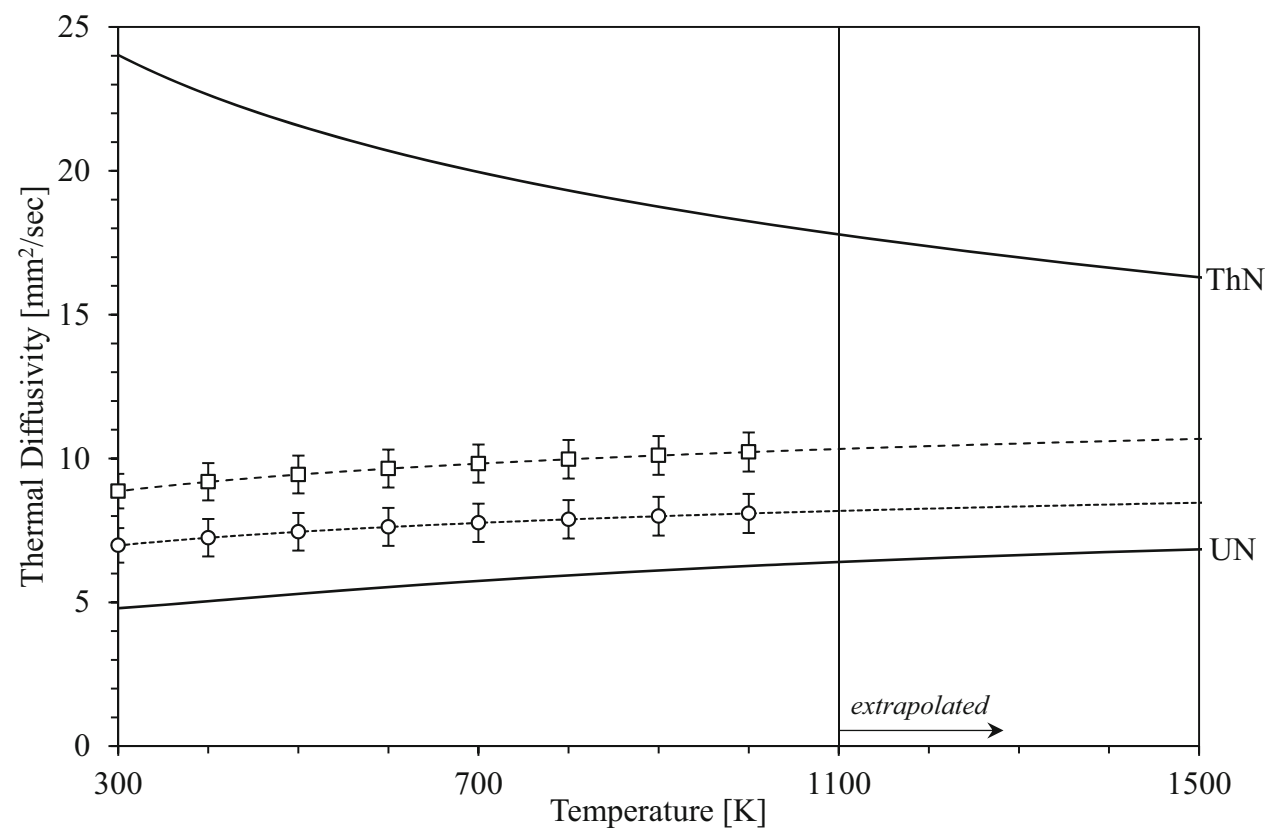

Fig. 6. Thermal diffusivity of $\left(\mathrm{Th}_{0.2} \mathrm{U}_{0.8}\right) \mathrm{N}$ (Circle) and $\left(\mathrm{Th}_{0.5} \mathrm{U}_{0.5}\right) \mathrm{N}$ (Square) plotted as a function of temperature. Theoretical prediction of the thermal diffusivity is given by the dashed lines and data for $\mathrm{UN}^{8}$ and $\mathrm{ThN}^{12}$ are plotted for reference.

similar porosity dependence. Interestingly, the thermal diffusivity of $\left(\mathrm{Th}_{0.5} \mathrm{U}_{0.5}\right) \mathrm{N}$ retains a positive slope, which is a characteristic feature of UN. The likely cause of this has been discussed previously as the result of the contribution of $5 \mathrm{f}$ electrons to thermal conduction in UN. However, estimation of the thermal diffusivity by rule-of-mixtures suggests that the thermal diffusivity should be essentially temperature invariant at this composition. However, this excessive electronic contribution to thermal diffusivity is consistent with the heat capacity data, which were also higher than expected and which exhibited a strong electronic component. This certainly merits further study of the electronic properties of UN as a function of dilution by other, soluble actinides.

\section{Thermal Conductivity}

The thermal conductivity of $\left(\mathrm{Th}_{0.2} \mathrm{U}_{0.8}\right) \mathrm{N}$ and $\left(\mathrm{Th}_{0.5} \mathrm{U}_{0.5}\right) \mathrm{N}$ have been calculated as a function of temperature and is plotted in Fig. 7. Porosity corrections, described in Refs. 14 and 15, are applied to both datasets. The porosity-corrected thermal conductivity is $\sim 10-15 \%$ lower than what is predicted from volume fraction calculations of each phase contribution to thermal conductivity. ${ }^{16}$ This may be due to minor oxygen impurity, which has been shown to cause significant degradation of thermal conductivity in both UN and in UN + PuN nitride mixtures. ${ }^{17,18}$ However, no apparent surface oxidation was observed prior to testing the samples. Carbon is a known possible contaminant within the LFA enclosure, and perhaps the preferential formation of thorium carbide on the surface of the samples could lead to a slight depression in the diffusivity data. However, measurements of the heat capacity and coefficient of thermal expansion of the mixed nitrides are in close agreement with theoretical predictions. Given that these methods are relatively insensitive to porosity, this result suggests that gross chemical impurities are not a dominant factor. More importantly, the accuracy of porosity correction models for thermal diffusivity is reduced with increasing porosity. Thus, given the porosity of the samples prepared for thermal diffusivity, the reported thermal conductivity should be viewed as a minimum bound, with the likely performance of these mixtures being slightly higher.

For low porosity $(P<10 \%)$, the porosity models show excellent agreement with empirical findings. This was the case in the measurement of thermophysical properties of UN and ThN ${ }^{8,12}$ In the limit of moderate porosity $(20 \%<P<10 \%)$, aberrations in pore distribution, size, and shape may test the core assumptions which define the range of applicability of the model. The LFA samples for the mixed nitrides fall into this range of porosity $(18 \%<P<16 \%)$. The assumptions of homogenously distributed, approximately spherical pores appear to hold for samples studied here, as demonstrated by Fig. 8, which consists of SEM images of a fracture cross-section and the sample surface in the case of a $\left(\mathrm{Th}_{0.5} \mathrm{U}_{0.5}\right) \mathrm{N}$ pellet for laser flash analysis ( $83 \%$ TD). The grain size ranges from 1 to $10 \mu \mathrm{m}$, with an average grain size around $5 \mu \mathrm{m}$. Larger void spaces in Fig. 8a are interpreted as missing grains removed during fracture, while the $<2-\mu \mathrm{m}$ voids are the pores. Pores are distributed 


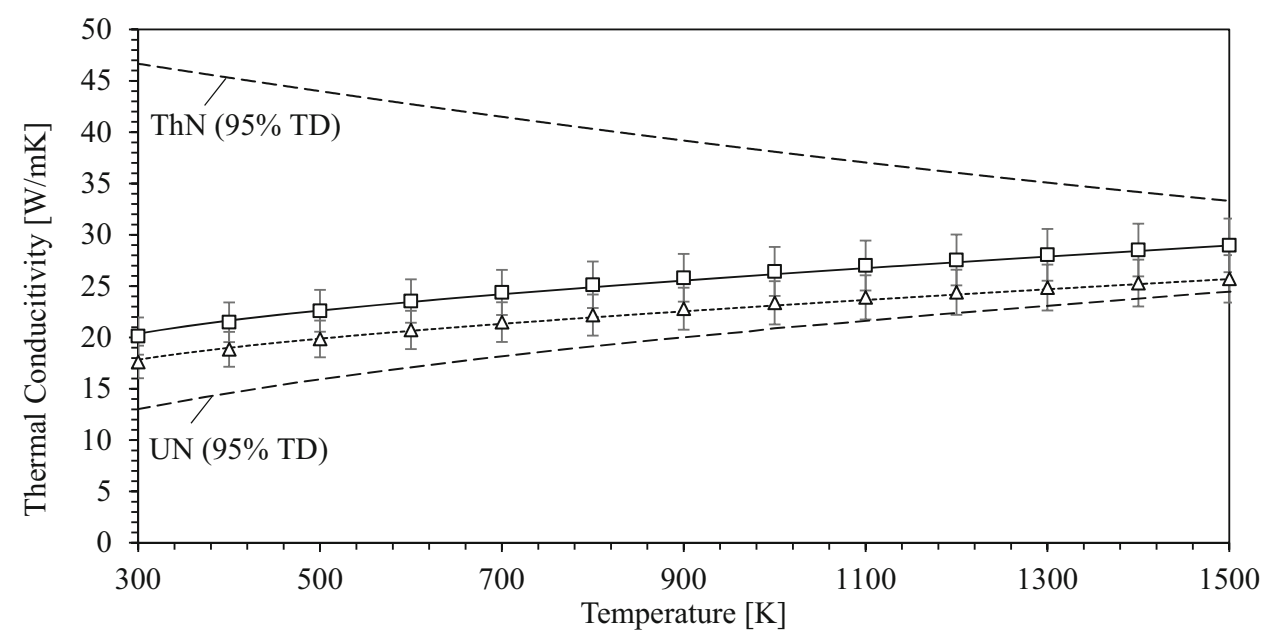

Fig. 7. Calculated thermal conductivity of $\left(\mathrm{Th}_{0.2} \mathrm{U}_{0.8}\right) \mathrm{N}$ (Triangle) and $\left(\mathrm{Th}_{0.5} \mathrm{U}_{0.5}\right) \mathrm{N}$ (Square). The thermal conductivity of pure ThN and UN have been included for comparison. ThN data are from Ref. 12 and the UN data are from Ref. 8.
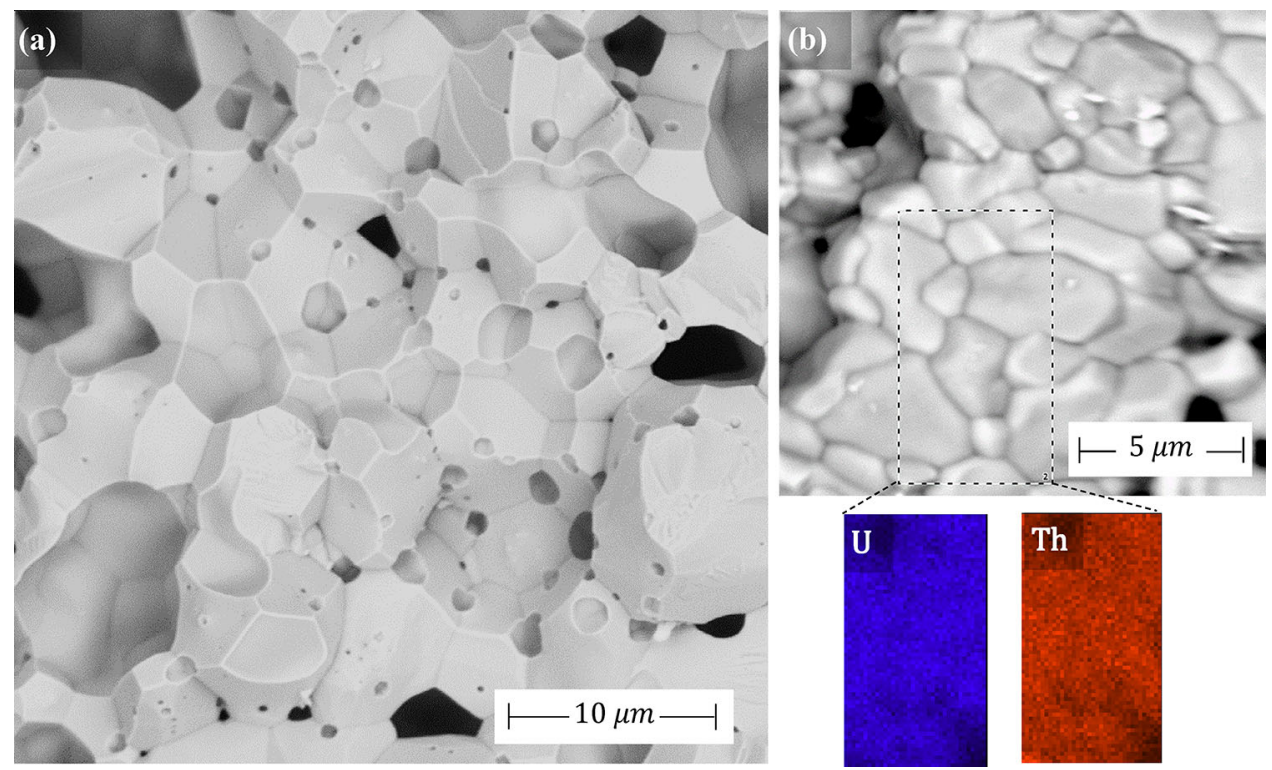

Fig. 8. Backscatter scanning electron micrographs of a $\left(\mathrm{Th}_{0.5} \mathrm{U}_{0.5}\right) \mathrm{N}$ pellet for laser flash analysis (83\% TD). The regions shown are: (a) a fracture surface across the cross-section of the sample, and (b) the surface of the sample, along with energy dispersive x-ray spectroscopy scans which show that $U$ and Th are homogeneously distributed.

along grain boundaries, and are especially prevalent at the intersection of grain boundaries. Fewer larger pores $(\sim 4-8 \mu \mathrm{m})$ are present in the sample. However, they do not appear to create interconnected channels of open porosity. Figure $8 b$ is accompanied with an energy dispersive x-ray spectroscopy scan, which shows the co-location of $U$ and Th signals, and the absence of any apparent discrete oxide or carbide species on the surface of the sample.

Error in the available porosity correction data and models is accounted for in the calculations of thermal conductivity in the form of conservative overestimation on the reported error bars $(\sim 9 \%)$. The essential trend and order of magnitude of the measured thermal conductivity remains apparent, and emphasizes the value of additional studies of the effects of porosity and oxygen impurities on the resultant thermal properties of ThN and mixtures of $\left(\mathrm{Th}_{\mathrm{x}} \mathrm{U}_{1-\mathrm{x}}\right) \mathrm{N}$, especially thermal diffusivity. It would appear that the high thermal conductivity of pure ThN may be a result of phononic transport in ThN, which is significantly diminished with additions of UN. It would be valuable to determine if dilute additions of UN in a matrix of ThN result in a proportional change in the measured thermal conductivity, especially in the range of $300-900^{\circ} \mathrm{C}$. The data reported for $\left(\mathrm{Th}_{0.2} \mathrm{U}_{0.8}\right) \mathrm{N}$ are in reasonable agreement with estimation by rule-of-mixtures. For the temperature range of $300-1500 \mathrm{~K}$, the equations 
of fit for the thermal conductivity of $\left(\mathrm{Th}_{0.2} \mathrm{U}_{0.8}\right) \mathrm{N}$ and $\left(\mathrm{Th}_{0.5} \mathrm{U}_{0.5}\right) \mathrm{N}$ are given by Eqs. 6 and 7 , respectively:

$$
\begin{aligned}
& \lambda_{\text {eff },\left(T h_{0.2} U_{0.8}\right) N}=4.61 \cdot T^{0.234} \\
& \lambda_{\text {eff },\left(T h_{0.5} U_{0.5) N}\right.}=5.54 \cdot T^{0.226}
\end{aligned}
$$

The porosity correction applied for the determination of $\lambda_{\text {eff }}$. is taken from Ref. 15 and has the functional form given by Eq. 8:

$$
\lambda_{\text {eff }}(x, T)=\lambda_{\text {empirical }}(T) \cdot \frac{1+\beta \cdot \gamma}{1-\gamma}
$$

where $\gamma$ is the porosity of the sample, and $\beta$ is a fitting factor and is usually on the order of 1-3. Fitting thermal diffusivity data for a measured porosity of $\gamma=0.18$, and assuming that the porosity dependence of $\left(\mathrm{Th}_{\mathrm{x}} \mathrm{U}_{1-\mathrm{x}}\right) \mathrm{N}$ is proportional to that of $\mathrm{UN}$ for compositions of $x \leq 0.5$, the fitting parameter $\beta$ is 2 for $x=0.2$ and 3.28 for $x=0.5$. Further study of the porosity dependence of the thermal conductivity of $\mathrm{ThN}$ and $\left(\mathrm{Th}_{\mathrm{x}} \mathrm{U}_{1-\mathrm{x}}\right) \mathrm{N}$ would reduce uncertainty in the reported thermal conductivity data. In similar studies, this model has been shown to work well in predicting the porosity dependence on $\left(\mathrm{U}_{\mathrm{x}} \mathrm{Pu}_{1-\mathrm{x}}\right) \mathrm{N}$ for compositions of $x=$ $0.8,0.65,0.4$, and 0.2 and porosities of $0.01 \leq \gamma \leq 0.20 .^{15,19-21}$

\section{Finite Element Modeling of Thermal Profile: A Case Study}

A simulation of the neutronic and thermal performance of $\left(\mathrm{Th}_{0.25} \mathrm{U}_{0.75}\right) \mathrm{N}$ fuel in a compact nuclear reactor was produced as a case study in order to demonstrate the proof of concept of this fuel form. Neutronic modeling was accomplished through Monte Carlo N-Particle Version 6.2 (MCNP-6); the cross-section of the model is presented in Fig. 1a, and an Abaqus FEA finite element model of a single hexagonal section of the compact core is shown in Fig. 1b. The basic design requirements are that this core would produce $10 \mathrm{MW}$ (thermal) for 10 years. The total fuel mass is $4572 \mathrm{Kg}$, and the initial ${ }^{235} \mathrm{U}$ enrichment is $19.5 \%$. The reactor operates in the fast spectrum, with heat removal by liquid $\mathrm{NaK}$. The energy density simulated by the neutronic model is an input parameter into the finite element model. The temperature distribution across the fuel and the coolant at startup are shown in Fig. 9. Given a boundary condition that the maximum coolant temperature does not exceed $700^{\circ} \mathrm{C}$, the $\Delta T$ from fuel centerline to coolant centerline is $\sim 60^{\circ} \mathrm{C}$. There is assumed to be no gap between the fuel and the structural assembly. Higher centerline temperatures would be expected if a coolant of lower thermal conductivity is used, or if a gap is present between the fuel and the cladding. The predicted centerline temperature of this fuel composition is

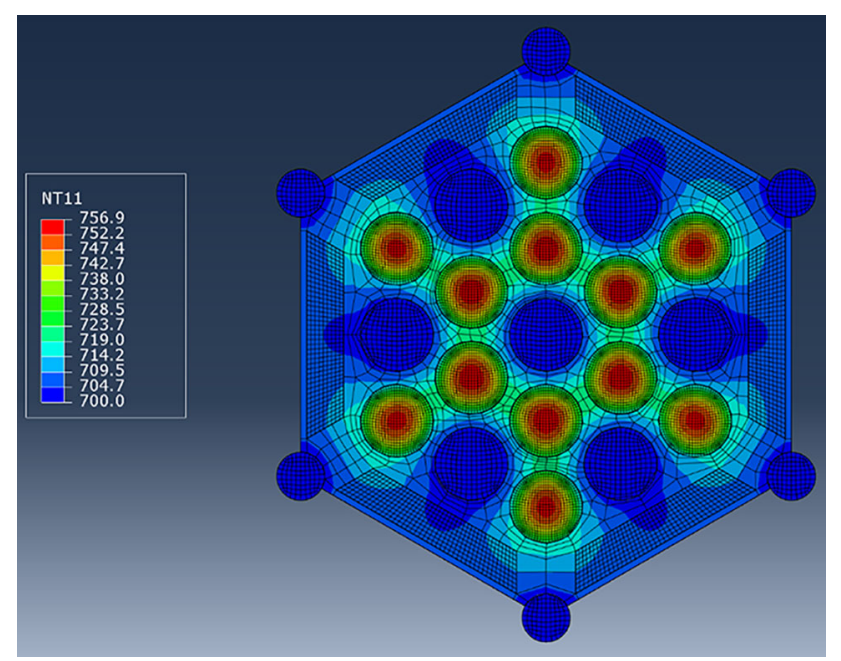

Fig. 9. Abaqus FEA model of the steady state thermal profile across the middle cross-section of the compact core. Temperature map is in units of ${ }^{\circ} \mathrm{C}$. The largest change in temperature across the fuel is less than $30^{\circ} \mathrm{C}$ (Color figure online).

more than $250 \mathrm{~K}$ less than what is expected from $\mathrm{UO}_{2}$ in this model. ${ }^{22}$

In Fig. 10, the thermal profile of a fuel bundle under the design basis failure of two adjacent heat pipes is shown. The greatest temperature difference established is $125^{\circ} \mathrm{C}$, with a maximum centerline temperature of $825^{\circ} \mathrm{C}$. These temperatures are well within the tolerable limits of the fuels and surrounding materials, based on melt points of core components and the boiling point of the coolant (NaK). Also shown is the maximum temperature reached by the two adjacent fuel rods. The relative change in reactivity from $756^{\circ} \mathrm{C}$ (steady-state) to $825^{\circ} \mathrm{C}$ (double heat pipe failure) would amount to a $\sim 0.01 \%$ decrease in reactivity, owing to the negative reactivity coefficient associated with this fuel form.

The experimental and modeling portions of this work drove a positive feedback loop. The Monte Carlo simulation outlined a useful fuel composition space, the Abaqus FEA model defined the high and low temperature limits, and experimental data served as input parameters to both. This case study serves to emphasize the application of the experimental research, and it demonstrates the feasibility of compact reactors based on a nitride fuel architecture.

\section{CONCLUSION}

The miscibility, lattice parameter, and thermophysical properties of $\left(\mathrm{Th}_{0.2} \mathrm{U}_{0.8}\right) \mathrm{N}$ and $\left(\mathrm{Th}_{0.5} \mathrm{U}_{0.5}\right) \mathrm{N}$ were studied and are discussed in comparison to the properties of pure UN and ThN. Following sintering, x-ray diffraction techniques were utilized to measure the lattice parameters of the as-sintered mixtures. These measurements, in conjunction with energy dispersive spectroscopy on the polished cross-section of the mixed nitride samples, verify 


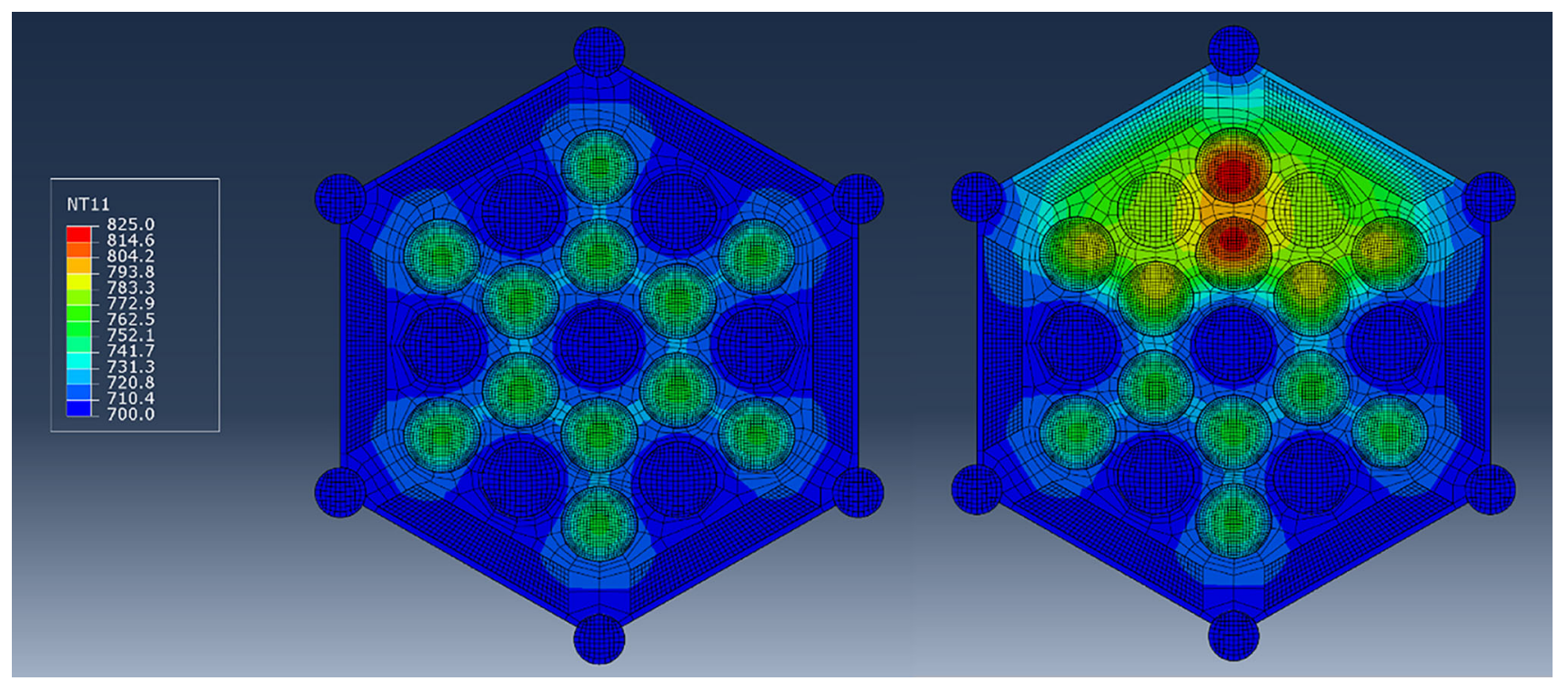

Fig. 10. A temperature-normalized Abaqus FEA model of (left) the steady-state thermal profile across the middle cross-section of the compact core, and (right) the thermal profile which results from a double heat pipe failure. Both images are normalized to the same temperature scale, which is in units of ${ }^{\circ} \mathrm{C}$ (Color figure online).

mutual solid solubility. The measured thermophysical properties of $\left(\mathrm{Th}_{0.2} \mathrm{U}_{0.8}\right) \mathrm{N}$ and $\left(\mathrm{Th}_{0.5} \mathrm{U}_{0.5}\right) \mathrm{N}$ as a function of temperature have been presented. The differential scanning calorimetry and dilatometry data, which are relatively insensitive to sample porosity, were found to be in close agreement with theoretical estimates from the rule-of-mixtures from data of pure UN and ThN. The effect of sample porosity was apparent in the thermal diffusivity data; however, conservative porosity corrections from models built around mixed UN and PuN fuels were applied to the data presented here and were shown to be consistent with the data expected from theoretical projections using the properties of ThN and UN. The thermal property measurements are considered the lower limit on the thermal performance of $\left(\mathrm{Th}_{0.2} \mathrm{U}_{0.8}\right) \mathrm{N}$ and $\left(\mathrm{Th}_{0.5} \mathrm{U}_{0.5}\right) \mathrm{N}$, given uncertainty introduced by sample porosity in thermal diffusivity measurements. The thermal expansion of the mixed nitride in the limit of dilute additions of ThN may deserve closer study in the future to determine the ratio of $\mathrm{ThN}$ to $\mathrm{UN}$ in order to optimize this fuel form from a thermomechanical perspective. This study is a first look towards the characterization of mixed $\left(\mathrm{Th}_{\mathrm{x}} \mathrm{U}_{1-\mathrm{x}}\right) \mathrm{N}$ fuels, and serves as a starting point to guide future studies. Additionally, the majority of the findings here suggest that the properties of mixed nitrides follow the rule-of-mixtures of the properties of the pure components. A comprehensive study of the effects of porosity on thermophysical and mechanical properties of these materials will allow mixed nitride fuels to be tailored to better suit the needs of the design requirements of the core. A case study of a compact reactor has been presented, and an Abaqus finite element model was used to determine the thermal profile associated with a fuel composition of $\left(\mathrm{Th}_{0.25} \mathrm{U}_{0.75}\right) \mathrm{N}$.

\section{ACKNOWLEDGEMENTS}

This work was supported by the U.S. Department of Energy, Office of Nuclear Energy Fuel Cycle Research and Development program. This work was also supported by the Department of Energy National Nuclear Security Administration under Award Number DENA000097 through the Nuclear Science and Security Consortium.

\section{CONFLICT OF INTEREST}

On behalf of all authors, the corresponding author states that there is no conflict of interest.

\section{OPEN ACCESS}

This article is licensed under a Creative Commons Attribution 4.0 International License, which permits use, sharing, adaptation, distribution and reproduction in any medium or format, as long as you give appropriate credit to the original author(s) and the source, provide a link to the Creative Commons licence, and indicate if changes were made. The images or other third party material in this article are included in the article's Creative Commons licence, unless indicated otherwise in a credit line to the material. If material is not included in the article's Creative Commons licence and your intended use is not permitted by statutory regulation or exceeds the permitted use, you will need to obtain permission directly from the copy- 
right holder. To view a copy of this licence, visit $h$ ttp://creativecommons.org/licenses/by/4.0/.

\section{REFERENCES}

1. J.W.L. Pang, W.J.L. Buyers, A. Chernatynskiy, M.D. Lumsden, B.C. Larson and S.R. Phillpot, Phys. Rev. Lett. 110(15), 157401 (2013).

2. S. Sheykhi and M. Payami, Iranian J. Sci. Technol. Trans. A 44(5), 1585 (2020).

3. H. Nakamura and M. Machida, J. Nucl. Mater. 519, 45 (2019).

4. Q. Yin and S.Y. Savrasov, Phys. Rev. Lett. 100, 225504 (2004).

5. V.I. Vybyvanets, M.L. Taubin, E.S. Solntseva, I.E. Galev, V.G. Baranov, A.V. Tenishev and O.V. Khomyakov, At. Energ. 117(4), 257 (2015).

6. T.R. Govindan Kutty, J. Banerjee and A. Kumar, Thermophysical Properties of Thoria-based Fuels (Springer, London, 2013).

7. N. Brown, M. Todosow and A. Cuadra, J. Nucl. Mater. 462, 26 (2015).

8. S. Hayes, J. Tomas and K. Peddicord, J. Nucl. Mater. 359(12), 17 (1990).

9. I.J. Hastings and R.D. MacDonald, J. Nucl. Mater. 126, 177 (1984).

10. H. Muta, K. Kurosaki, M. Uno and S. Yamanaka, J. Mater. Sci. 43, 6429 (2008).
11. M. Valter, Thermal Conductivity of Uranium Mononitride, Linkoping University. LITH-IFM-A-EX-15/3096-SE (2015).

12. S.S. Parker, J.T. White, P. Hosemann and A.T. Nelson, J. Nucl. Mater. 526, 151760 (2019).

13. J.T. Dunwoody, C.R. Stanek, K.J. McClellan, S.L. Voit, H.M. Volz and R.R. Hickman, American Nuclear Society Conference (2007)

14. T. Kikchi, T. Takahashi and S. Nasu, J. Nucl. Mater. 45, 284 (1973).

15. G.P. Marino, J. Nucl. Mater. 38(2), 178 (1971).

16. J.P. Gorton, B.S. Collins, A.T. Nelson and N.R. Brown, Nucl. Eng. Des. 355(1), 110317 (2019).

17. Y. Arai, M. Morihira and T. Ohmichi, J. Nucl. Mater. 202, 70 (1993).

18. A.A. Bauer, Reactor Technol. 15(2), 87 (1972).

19. R.B. Kotel'nikov, S.N. Bashlykov and A.N. Kashtanov, High-Temperature Nuclear Fuel, 2nd edn. (Atomizdat, Moscow, 1978) (in Russian).

20. S.B. Ross, M.S. El-Genk and R.B. Matthews, J. Nucl. Mater. 151(3), 318 (1988).

21. C. Ganguly, P. Hegde and A. Sengupta, J. Nucl. Mater. 178, 234 (1991).

22. D.A.P. Palma, A.Z. Mesquita, F.C. Marinho and M.S. Rocha, J. Energy Power Eng. 8(6), 1054 (2014).

Publisher's Note Springer Nature remains neutral with regard to jurisdictional claims in published maps and institutional affiliations. 